\title{
e-PBL Model Development for Computer Learning Systems
}

\author{
Kil Hong Joo ${ }^{1}$ and Nam Hun Park ${ }^{2}$ \\ ${ }^{1}$ Dept. Of Computer Education, Gyeongin National University of Education, \\ San 6-8 Seoksudong Manangu Anyangsi, Gyeonggi, Korea, 430-040 \\ ${ }^{2}$ Dept. Of Computer Science, Anyang University, 102 Samsungli, \\ Buleunmyun, Ganghwagun, Incheon, Korea, 417-833 \\ ${ }^{1}$ khjoo@ginue.ac.kr, ${ }^{2}$ nmhnpark@anyang.ac.kr
}

\begin{abstract}
In this paper, an e-PBL model and problems for an effective computer education were developed and applied for information literacy education of computer in elementary schools. In order to progress an effective e-PBL class, a support learning system including an e-mentoring and a web discussion function has to be built. In this paper, we developed the e-PBL teaching model for information literacy education of computer in elementary schools and inspected its effectiveness and validity. In order to resolve the research questions, class elements were extracted from an analysis of the prior research in the e$P B L$. The class elements in the e-PBL were analyzed to six elements of the 'unstructured practical problem', 'learner-centered problem', 'process reflected problem', 'cooperative learning reflected problem', 'on-line learning support system', and 'e-mentoring'. The e$P B L$ model was designed based on the extracted elements. The model developed by the present research added an on-line element and an e-mentoring element to the conventional $P B L$ model. The main learning steps were given by 6 steps and it was characterized that the e-PAS system (Learning support system) and e-mentoring were used. The main steps include an introducing step, a problem proposing step, a problem solving plan step, a problem solving step, a result proposing and presenting step, a class finishing step, and an evaluation step. For the successful e-PBL teaching in elementary education of computer, the learning system that has the various learning strategies and support systems and interacts teachers with learners frequently will ought to be built.
\end{abstract}

Keywords: e-learning, learning system, PBL, Mentoring

\section{Introduction}

According to the needs of the times, there has been a demand for a process for redefining and reproducing existing information as well as a process for producing and distributing new information. In the information society, the production of the new information and the reproduction of the existing information have been recognized as important assets and have demanded consistent changes in daily life. Now, the ability to acquire and utilize Information and Communication Technology has become a survival strategy beyond personal development. Due to such a paradigm change, the conventional teaching and learning method, which simply aims at the delivery and acquisition of knowledge, has become inappropriate to increase the self-directed problem solving ability required in daily life.

Therefore, 'the improvement in the self-directed learning ability to become adjusted to the information society' and 'the practice of a learner centered education adequate to the students' aptitudes, abilities, and careers' have been emphasized. Also, the ability to use studied contents to solve daily life problems has been increased to improve self-directed adaptability in the real world. This agrees with an argument of Guzdial, who said "Since 
the variety of abilities required in the information society cannot be separated from actual circumstances, education must be accomplished in that circumstance" [1].

Under such direction, a Problem Based Learning (hereinafter, referred to as PBL), in which learning is accomplished in the center of a very 'complicated' and 'unstructured' 'task' on the basis of a certain 'circumstance', is rising as a new alternative plan [2,3]. The PBL based on the epistemology of constructivism is an educational approach constructed by a curriculum and teaching in the center of the problematic situation in actual life. Accordingly, it is a type of learning which allows students to improve their critical thinking ability and cooperation capability while solving the problem. If an ultimate objective of education is not only to acquire knowledge but to improve the problem solving ability in daily life, it can be said that the PBL, in which learning is accomplished by solving an unstructured task starting from a certain specific problem based on a particular circumstance, is the most adequate teaching-learning model [4].

Problem based learning, which has been used and studied in various fields, has many characteristics besides concepts. Barrow(1996) argues that the learning in the PBL is achieved by students in the center and occurs in small groups. A teacher is an advisor or a guide. Further, the teacher organizes 'problems' as a stimulus and a core to learn and the problems are means for developing the problem solving ability. In addition, he said that new information has to be acquired by the self directed learning.

In order to perform an e-PBL research, the present paper chose the following research questions. First, e-PBL elements applicable in an effective computer education are extracted from an analysis of a prior PBL research adapted in other subjects. Second, an ePBL learning model and problems, which can be used in the information communication technical education at the sixth grade level of an elementary school, are developed and applied. Finally, application results are analyzed to verify the effectiveness and validity of the e-PBL learning model and the e-PBL learning problems.

\section{Designing and Building an e-PAS System to Support e-PBL}

Currently, the teaching time assigned for information and communication technology education at an elementary school is one hour per week on average and there is a physical limitation to achieving the learning objectives of computer education. Accordingly, the present paper proposes an e-PBL model of blended learning, in which conventional offline learning and on-line learning are blended. The e-PBL learning model is based on the PBL model of Barrows and Myers(1993). The e-PBL starts from an unstructured practical problem and sets great importance on the problem solving process by cooperative learning and self directed learning. Here, cooperative learning and self directed learning include dividing each student's roles required to solve the problem in small groups; solving the given parts; and combining each student's opinions in the groups. Analysis of existing studies show that the e-PBL includes an 'unstructured practical problem', 'learner-centered learning', 'processes', and 'cooperative learning' in Table $1[5,6,7]$.

The e-PAS system is a system to support the on-line part in the e-PBL. When categorizing this system into the types of usage by the e-learning contents, this system is formed of a WBI/HTmL type and a C\&A/library type and includes a web discussion function, a bulletin board function, an e-mentoring web discussion function, and a note function $[8,9,10]$.

In one aspect of the content of the e-PBL, computer literacy education is the education of information and communication technology itself and it emphasizes acquiring professional concepts and practical skills to solve the problem, rather than searching for the information and knowledge. Therefore, the individual learning, which is a process for acquiring skills by the learners themselves, is emphasized as well and the basic concepts or learning skills required to solve the problem should be accurately instructed by the teacher. In addition, in the present paper, the cooperative learning process, usually performed as a 
task divided type in the PBL in general, is performed to exchange each other's opinions and knowledge on-line.

Table 1. Characteristics of the PBL

\begin{tabular}{|c|c|c|}
\hline \multicolumn{2}{|c|}{ Categories } & Content \\
\hline \multicolumn{2}{|c|}{ Problem } & $\begin{array}{l}\text {-Unstructured problem } \\
\text {-complicated and practical context problem }\end{array}$ \\
\hline \multirow{2}{*}{$\begin{array}{c}\text { Roles of teacher } \\
\text { and students }\end{array}$} & teacher & $\begin{array}{l}\text {-cognitively helping and coaching } \\
\text {-proposing a problematic situation } \\
\text {-modeling, helping, elaborating } \\
\text {-evaluating learning processes and results }\end{array}$ \\
\hline & students & $\begin{array}{l}\text {-active participation and problem solving } \\
\text {-organizing knowledge in the practical context } \\
\text {-problem solving on the basis of knowledge and } \\
\text { experience }\end{array}$ \\
\hline \multicolumn{2}{|c|}{$\begin{array}{c}\text { Teaching and learning } \\
\text { method }\end{array}$} & $\begin{array}{l}\text {-interaction between teaching and learning } \\
\text { (teacher and students, student-student, } \\
\text { student-material) } \\
\text {-Self directed learning (learning objectives setting, } \\
\text { self evaluation) } \\
\text {-cooperative learning(dividing roles, sharing } \\
\text { knowledge, peer evaluation) }\end{array}$ \\
\hline \multicolumn{2}{|c|}{ evaluation } & $\begin{array}{l}\text {-portfolio suitable for solving the problem } \\
\text {-Worksheets demanding practical problem solving } \\
\text {-ended evaluation observation, reflection note }\end{array}$ \\
\hline
\end{tabular}

The e-PBL proposed in the present paper emphasizes the cooperative learning and the self directed learning in the aspect of studying. After deciding each student's roles in solving the problem, each one of the group members solves the given part, exchanges their opinions in their groups, and uses them. In addition, in one aspect of the learning content, it is more focused on acquiring professional concepts and utilization abilities rather than searching for the information and the knowledge by information and communication technology literacy education so as to solve the problem. Accordingly, in the present paper, the e-PBL model as described in Figure 1 is proposed.

The e-PBL model proposed in the present paper includes six steps: introducing; proposing a problem; planning to solve the problem; solving the problem; proposing and presenting the results; and finishing the learning and evaluating. In the class developing step, which is a specific step in the introducing step, the e-PBL is introduced and the learning atmosphere is formed, and then the processes of the learning and roles of the teacher and the students are introduced. The proposing a problem step specifically includes a problem setting step and a group making step. In the problem setting step, the given 
problem is analyzed by using the e-PAS system and the kinds and types of tasks, due dates, and important learning elements are explained.

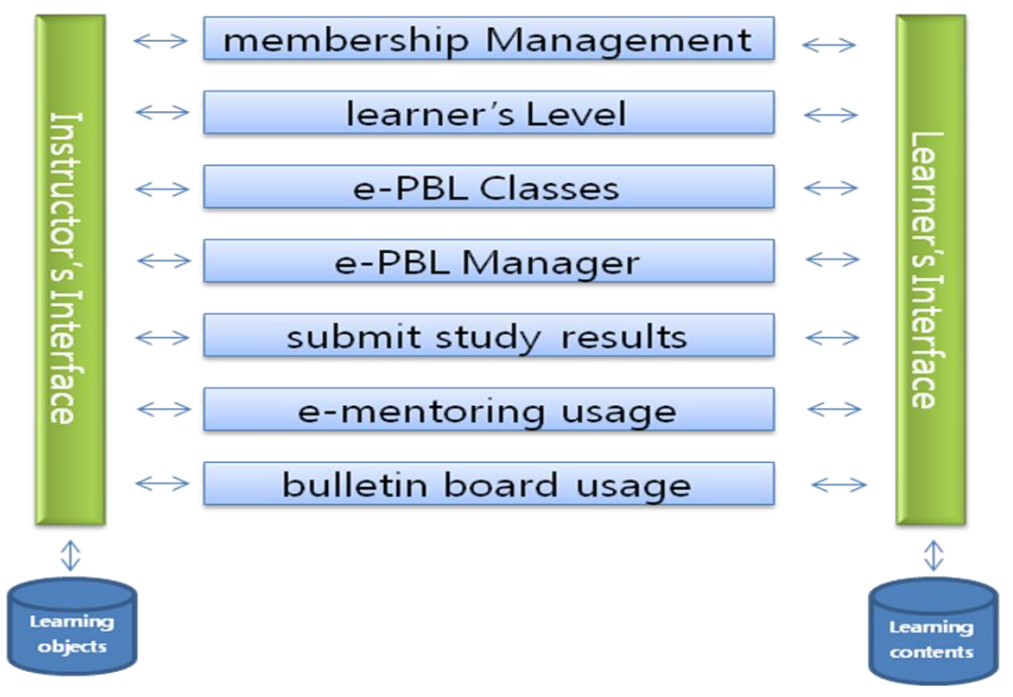

Figure 1. e-PAS Module

The planning step for solving the problem specifically includes setting learning objectives, group discussions, dividing roles, and setting individual tasks. In this step, the objectives are specified and then things that are already known, things that need to be known to solve the problem, and available ICT skills are specifically categorized.

The problem solving step specifically includes an activity to solve the problem and a step for verifying interim results. The activity to solve the problem includes exchanging group members' opinions and exchanging information. Any part unsolved by the learner in this process is resolved by help from a learning facilitator (teacher) and classmates by using the e-PAS system. The step for proposing and presenting the results specifically includes arranging and developing the final results, and proposing and presenting the results. That is, learners practice how to write a report and present the final results.

Finally, the step for finishing the learning and evaluating includes a discussion and evaluation. That is, the learning processes are examined and a generalization is made from the self evaluation, the group members' evaluation, and the evaluation between groups.

In the present paper, a web-based e-PAS system (e-PBL Assistance System) to support an efficient e-PBL class is proposed. The present system includes processes for the e-PBL cooperative learning and group learning, an instructor module for managing information, and a learner module. The students can register themselves, manage learning, collect information, perform mentoring, and conduct web discussions through the learner's interface and the instructor can manage the information through the instructor's interface.

This system supports the following roles. First, the system provides the learners with appropriate learning activities on-line. Second, the system is used as a means of collecting and saving learning materials for class development and submitting the study results. Third, the system provides the e-mentoring activity concerning the learning method, the problem solving strategies, and the structure of the problem which are made in the learning activity. Fourth, the system takes the role of sharing and developing the learning content by exchanging information between the students. Figure 2 is a general configuration of the e-PAS system proposed in the present paper.

The e-PAS system is the e-PBL supporting system and overcomes the limitations of time and space which are the weak points of the PBL and includes an on-line mentoring module. 
The operator module functions to manage users through the administrator interface, learning objects, learning content, memberships by using message management data base, levels, the e-PBL learning objects, the e-PBL Manager, the study results, the mentoring, the web discussions, the bulletin, and the note message. The instructor can deliver content to all of the students by using the bulletin and can advise the individual students by using the note function.

- Membership and information management: The students can participate in the learning system by signing up and can manage personal information.

- Level management: The levels of learners are managed. The levels are classified by the roles in groups, the utilization of the e-PAS system, and the study levels and the usage of the library is limited depending on the levels.

- e-PBL learning objects: The origins of e-PBL learning, the process of learning, the introduction of the cooperative learning, and relevant web sites are studied.

- e-PBL Manager: the subject, the objectives, and methods of the e-PBL are presented and the submission date of the study results, the methods, and the study group configuration are introduced. In addition, the operation method of the e-PAS system is explained.

- submit the study results: the bulletin board where the intermediate study results and the final study results are submitted functions with the adding of comments for the study results.

- e-mentoring usage: E-mentoring can be performed by using the bulletin board, and the note. Basically, all users can open the page. If you do not want to disclose the information of the mentoring, you can lock or add comments.

- Bulletin board usage: A variety of bulletin boards for this system can be made and changed depending on the purpose, and the contents thereof can be saved by using a classifying function.

- Web Discussion: The problems generated in the learning progress and personal opinions about sharing data can be argued at liberty.

- Note and message usage: an individual can receive the note and the message.

\section{Experimental Results}

Since the e-PBL has the characteristics of being conducted both on-line and off-line, the principles for developing the problem were made based on the characteristics of the environment of two and the processes of selecting a topic, presenting the learning objectives, the outline, writing scenarios, and the verification were performed. Therefore, in the present paper, the following principles were applied to develop the e-PBL problem. First, a problem was selected which can reflect the students' real-life or a variety of experiences. Second, the problem should have an unstructured characteristic which can be solved by using knowledge, information and ideas. Third, cooperation with the school education curriculum should be considered.

To verify the effectiveness of this paper, 120 students from three classes of sixth graders at oo elementary school in Pyongtaek, Gyeong-gi province were chosen and homogeneity tests and ICT usage skill tests were performed. Therefore, as the learning topic adapted to the present paper, three subjects of a 'presentation', 'information world to be shared with', and 'various comprehensive activities' in the information and communication education curriculum of the elementary sixth grades were selected and the objectives for each area was set.

The present research was conducted by teaching the contents from 'Happy computer 6 grade' published by Gyeong-gi Province education board, based on the information and communication technology education curriculum for elementary schools. Since the e-PBL can be performed both on-line and off-line, the principles for developing the problem were made based on the characteristics of the 
environment and the processes of selecting a topic, presenting the learning objectives, the outline, the writing scenarios, and the verification were performed. By considering the characteristics of the learning, the principles for developing the learning problem were developed, first, to reflect students' real life and various experiences, second, to reflect the nature of the unstructured characteristic to solve the problem by using overall knowledge, information, and thoughts, and third, to connect the information and communication technology education to the school curriculum. Based on those principles, three lessons of making a presentation, the world of sharing information, and various activities were selected for the sixth graders' discretionary activities.

To verify the results of this research, changes in the information acquiring ability before or after being subjected to the e-PBL were tested. In addition, in order to verify the effects of the learning model, the group evaluation, the evaluation in groups, and the evaluation between groups were conducted. As a means of verifying the information acquiring ability, there was used a certificate evaluation of computer application ability, a computer pre-interest test, and a practical examination. To verify the effects of the e-PBL problem and the learning model, an evaluation of the learning process and an evaluation of the results were conducted. In the evaluation process, an evaluation for groups' or individuals' attitudes to participation was conducted, and, in the results evaluation, an evaluation of the creative learning ability, an evaluation of the self directed learning ability, and an evaluation of value and attitude were conducted. To verify the effects of the e-PBL model, a progress evaluation and a result evaluation were conducted. As for the progress evaluation, the evaluation in groups and the evaluation between groups were conducted and as for the result evaluation, the evaluation of the creative learning ability, the evaluation of the self directed learning ability, and the evaluation of value and attitude were conducted.

In the evaluation of the creative learning ability, elements such as the ability to recognize the problem, the ability to understand the problem, the ability to plan for solving the problem, the ability to research, the ability to propose and present a resolution, and the ability to adapt in real life were considered. The interest and curiosity for the learning and the understanding of the problem were evaluated to determine the ability to recognize the problem and individual resolvability and creative thoughts were evaluated to determine the plan to resolve the problem. The cooperative learning ability, the e-PAS usability, the information usability, and the researching ability were evaluated to determine the researching ability; the ementoring usability was evaluated to determine the ability to propose and present the resolution; and the e-mentoring usability in real life was evaluated to determine utilization in real life. The main elements in the self directed learning ability evaluation (self evaluation) include spontaneity for solving the problem, learning desire and motivation, responsibility for solving the problem, and satisfaction toward the e-PBL class. The researching ability to solve the problem and the pre-recognition ability of the e-PBL problem were examined to determine the spontaneity to solve the problem; and the e-PBL interest and the learning tasks suitable for the level were determined for the learning desire and motivation. The discussion activity, the rational judgment, and the information sharing activity were evaluated to determine the responsibility for solving the problem; and participation in the learning activity, learning interest, acquiring skills for solving the problem, and the valuable information activity were evaluated to determine the e-PBL class satisfaction. Finally, groups' learning results with regard to problems, presentation attitude, enthusiasm in the class, and listening attitude to the presentation are evaluated to determine the value and the attitude.

To see an improvement in the creative learning ability after adapting the e-PBL model, a survey was conducted for the entire class. Figure 2 shows the percentage of students by 
three groups of a higher group, a medium group, and a lower group. As a result of the survey, the problem recognition ability was $61.5 \%$ in the higher group, $34.7 \%$ in the medium group, and $3.4 \%$ in the lower group and the problem solving plan ability was $40.9 \%$ in the higher group, $48.4 \%$ in the medium group, and $2.5 \%$ in the lower group. In addition, the researching ability was $44.3 \%$ in the higher group, $51.9 \%$ in the medium group, and $3.8 \%$ in the lower group and the problem solving and presenting ability was $56.4 \%$ in the higher group, $40.1 \%$ in the medium group, and $3.5 \%$ in the lower group. Finally, the generalization ability was $52.9 \%$ in the higher group, $48.4 \%$ in the medium group, and $4.1 \%$ in the lower group. According to an analysis of the elements of the creative learning, since many students responded in the higher group and the medium group, it can be said that the e-PBL class was effective in improving the creative learning ability.

In the problem recognition ability by tasks in the e-PBL, the higher group showed $67 \%$ in 'introducing traditional culture' and $62 \%$ in 'creating a yearbook' of question no. 3 , thus higher than an average of $61 \%$. In case of 'resolution material making of an environmental problem', many students responded for the 'medium group' rather than the 'higher group'. An analysis of the results shows that the students can collect various learning resources during the e-PBL class and they showed higher interest, curiosity, and problem understanding to the learning enabling the e-mentoring, which is effective between the students, the teacher, and the groups.

A verification result of the problem solving plan ability shows that an average of $97.5 \%$ by tasks made an effective plan and studied in the e-PBL problem solving, which is developed by the present paper. An analysis of the improvement in the researching ability shows that the sum of the 'higher group' and the 'medium group' is over $96 \%$, thus showing a positive reaction from many students. Therefore, it can be confirmed that the ePBL problems, made by the present paper, were effective for the cooperative learning ability, the information usage, and the e-PAS usage. An analysis result of the level of improvement in the solution proposal and the presentation ability confirmed that an average $96 \%$ of the respondents showed a positive reaction. Therefore, it can be confirmed that the e-PBL problems, made by the present paper, were exceedingly effective.

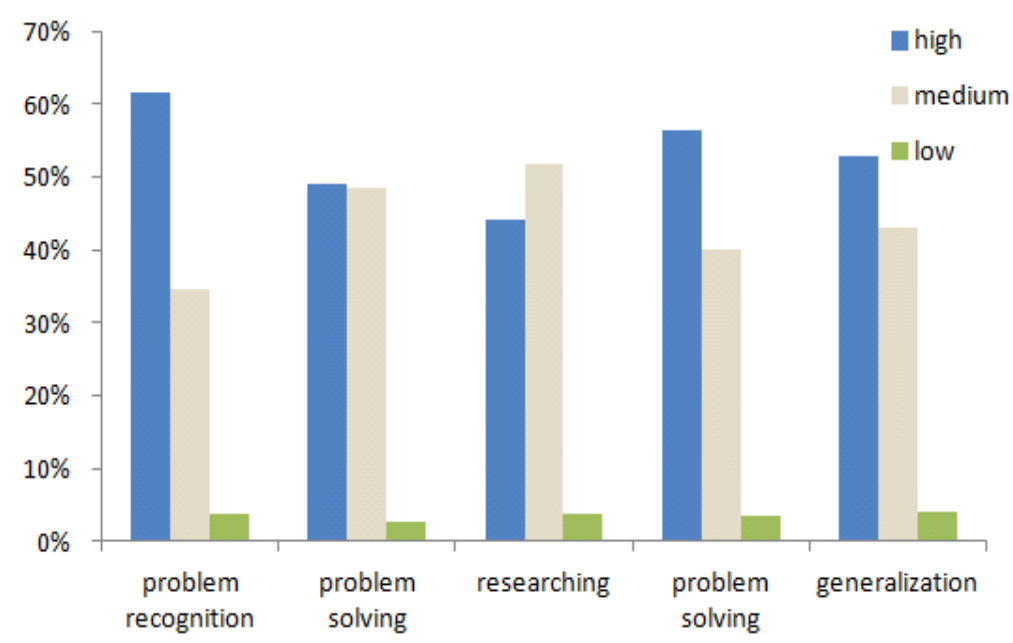

Figure 2. Analysis of Creative Learning Ability in e-PBL

The analysis result of responses for applications of the class and the development problem in real life showed that the students of the average $96 \%$ responded that the class and the development problem were helpful in real life. This confirms that the e-PBL model 
and the learning problems which are developed and applied in the present paper were actually effective to solve issues in computer education in real life.

\section{Conclusion}

An e-PBL model and problems for an effective computer education were developed and applied in the present research. As a result, the following conclusions were obtained. In order to progress an effective e-PBL class, a support learning system including an e-mentoring and a web discussion function has to be built. In addition, the e-PBL adapting problems should be an 'unstructured practical problem', 'learner-centered problem', 'process reflected problem', and 'cooperative learning reflected problem', which are directly involved in real life. Finally, the e-PBL problem and the model were effective to improve the students' information literacy ability. The results of the e-PBL model and the learning problems, which are proposed in the present research, can be changed depending on the support system, guages of the students, environment, and learning content. Therefore, the e-PBL model, the problems, and the support system, which reflect various learning environments, contents, ages, and levels of the students, should be continuously studied and developed. Also, class satisfaction, the ability to perform individual tasks, the group learning ability, mentoring usage, and usage satisfaction should be studied by applying a variety of analysis techniques.

Through this research, an effective e-PBL model and problems for elementary education were developed and applied. As a result, the present paper aims to verify changes in creative learning ability, self directed learning ability, and information literacy ability of the e-PBL model and the developed problems in computer education and the effectiveness and validity of the learning model. In order to achieve such a research objective, the following research questions were selected. First, a prior research of a PBL adapted in other subjects is analyzed to extract the e-PBL elements which can be adapted in elementary computer education. Second, the e-PBL model and the problems, which can be adapted in information and communication technology education at sixth grade level, are developed and applied. Third, the results are analyzed and the effectiveness and validity of the e-PBL and the problems in creative learning ability and self directed learning ability are verified.

As a measurement tool for the information and communication literacy ability, 25 questions developed at the headquarters of the Korea computer literacy certification were used and the fields of the evaluation include understanding and ethics of information, a memory device, understanding of hardware, use of an operating system, understanding and the use of a utility, the use of a word processor, understanding of a network, understanding of a web browser, and understanding and the use of the internet. The evaluation elements of the creative learning ability and the self directed learning ability were reflected and extracted from the e-PBL elements of 'Theory and Practice of problem based learning' by Cho, Yeon-Soon (2006). The evaluation elements of the creative learning ability used in the present paper were the problem recognition ability, the problem solving plan, the researching ability, the proposing and presenting ability for the resolution, and the e-PBL class satisfaction. In addition, as the self directed learning ability, the spontaneity to the problem solving, the learning enthusiasm and motivation, the responsibility to the problem solving, and the e-PBL class satisfaction. In order to adapt the e-PBL model, 120 students from three classes of sixth graders at $\mathrm{H}$ elementary school in Pyongtaek, Gyeong-gi province were chosen and experimental classes were conducted. As the learning contents, 'presentation', 'information world shared with each other', and 'various information activities' were selected based on the contents of 'fun computer sixth grader' published by Gyeong-gi province education board. The off-line classes were made of 4 periods and 12 periods were conducted in total. In order to perform successful classes, the e-PAS learning 
support system was operated and the e-mentoring system was actively used during the class. The e-PAS system consists of an operator module and a learner module and the online class is the core system.

As a result of developing and adapting the e-PBL model and the problems, the following conclusion was obtained. First, the e-PBL computer is an effective learning model to improve students' information literacy achievement. Particularly, it is more effective for the students of a higher level than the students of a lower level. Second, the e-PBL model is an effective learning model to increase the creative learning ability and the self directed learning ability of the students. Third, in order to perform an effective e-PBL class, a support learning system including an e-mentoring and a web discussion function has to be built. Fourth, the e-PBL adapting problems have to be the types where the 'unstructured practical problem', 'learner-centered problem', 'process reflected problem', 'cooperative learning reflected problem', 'on-line learning support system', and 'e-mentoring' are reflected.

\section{Acknowledgements}

This research was supported by Basic Science Research Program through the National Research Foundation of Korea(NRF) funded by the Ministry of Education, Science Technology(NRF-2014R1A1A1008524).

\section{References}

[1] H. Barrow, "Practice-based learning", I1: Southern Illinois University School of Medicine, (1994).

[2] T. Duffy and J. Kirkley, "Learner-centered theory and practice in distance education : Cases fron higher education", NJ : Lawrence Erlbaum Associates, Inc., (2003).

[3] H.S. Barrows and A.C. Myers, "Problem-Based Learning in Secondary Schools", Unpublished monograph, Springfield, IL: poblem-Based Learning Institute, Lanphier High School and Southern Illinois University Medical School, (1993).

[4] M. Guzdial, "Technological Support for Project- Based Learning”, Yearbook Association for Supervision and Curriculmu Development, (1998).

[5] N. Phumeechanya and P. Wannapiron, "Ubiquitous scaffold learning nvironment using problem-based learning to enhance problem-solving skills and context awareness", The Computing Research Repository, (2014).

[6] E. Tambouris, M. Zotou and K. A. Tarabanis, "Towards designing congnitively-enriched project-oriented courses within a blended problem-based learning context", Education and Information Technologies, (2014) vol. 19, no. 1.

[7] V. Ribaud, P. Saliou, "The cost of problem-based learning: An example in information systems engineering", CSSEE\&T, (2013); San Francisco, CA, USA.

[8] R. S. Hongxiu, "A proposed scale for measuring e-service quality", International Journal of u- and eservice, science and technology, (2009), vol. 2, no. 1.

[9] N. Arman, "E-learning materials development: applying and implementing software reuse principles and granularity levels in the small", International Journal of u- and e-service, science and technology, (2009), vol. 3 , no. 2 .

[10] J. Lee, J.-H. Kim, "Development and Analysis of web-based discussion system for elementary school students", International Journal of u- and e-service, science and technology, vol. 5, no. 3, (2012). 


\section{Authors}

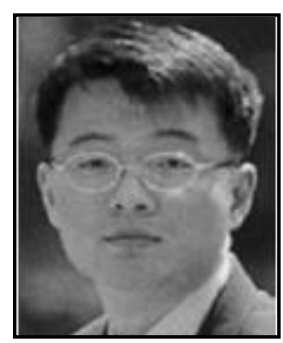

Nam Hun Park, he received the B.S., M.S. and Ph.D. degree in Computer Science from Yonsei University, Seoul, Korea, in 2000, 2002 and 2007. He was a post-Ph.D. at the Department of Computer Science, Worcester Polytech Institute, Worcester, MA. $\mathrm{He}$ is currently a professor of Department of Computer Science at Anyang University, Korea. His current interests include mining data streams.

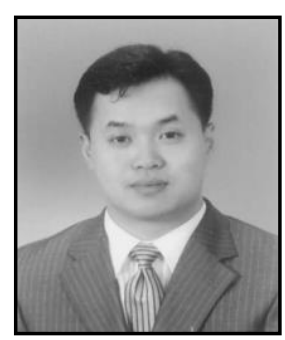

Kil Hong Joo, he received the M.S. and Ph.D. degree in Computer Science from Yonsei University, Seoul, Korea, in 2000 and 2004. He is currently a professor of Department of Computer Education at Gyeongin National University of Education, Korea. His current interests include mining data streams, data analysis and smart learning. 